\title{
Femoral hypoplasia- Unusual Facies Syndrome or Femoral Facial Syndrome with Radioulnar Synostosis
}

\author{
BEGUM SHARIFUN NAHER
}

\begin{abstract}
Introduction:
The femoral hypoplasia- unusual facies syndrome (FH/ UFS); also known as femoral facial syndrome (FFS) encompasses a spectrum of anomalies that include bilateral femoral hypoplasia and craniofacial dysmorphism with cleft palate, thin upper lip, micrognathia, flat philtrum, short nose with broad tip and upslanting palpebral fissure..$^{1,2}$ Other frequent findings include hypoplasia of fibulae, club foot, lumbar spine and pelvic anomalies. Systemic anomalies like cardiovascular and genitourinary anomalies may be associated with limb defects. This disorder was first discovered by Daentt et $\mathrm{al}^{3}$ in 1975 and is more common in females. ${ }^{1}$ The femoral hypoplasia- unusual facies syndrome is a rarely considered clinical entity that has a strong association with maternal diabetes. ${ }^{4}$

Etiopathogenesis of the syndrome remains unknown ${ }^{5}$. Majority of the cases described have been sporadic. We describe here a case with (FH/UFS)- a rare finding like distal radioulnar synostosis and separation of proximal ends of radius and ulna.
\end{abstract}

\section{Case report}

A four day old female baby $3^{\text {rd }}$ issue of nonconsanguinous parents admitted on 9th January, 2013 with the complaints of short and bowed lower limbs. Mother Jhumur 28 year old was normotensive but diabetic. She was on regular antenatal check up. Her diabetes was diagnosed before conception and was treated with insulin. She had fever for 5 days during her mid trimester when she received homeopathic treatment. Otherwise her pregnancy was uneventful up to term . Then she delivered a female baby by normal vaginal delivery at Mitford hospital .There was rupture of membrane 17 hour before delivery. Duration of delivery was 6 hour. Baby cried immediately after birth. There was no history of taking any offending drug during pregnancy. On examination appearance was dismorphic. Micrognathia, flat philtrum, small pinched nose (Fig.-1) and cleft palate was present. Anterior fontanel was normal. Skin color was pink.

Correspondence: Dr. Begum Sharifun Naher, Associate Professor, Department of Neonatology, SSMC, Mitford, Dhaka, E-mail: dr.sharifun_nahar@yahoo.com

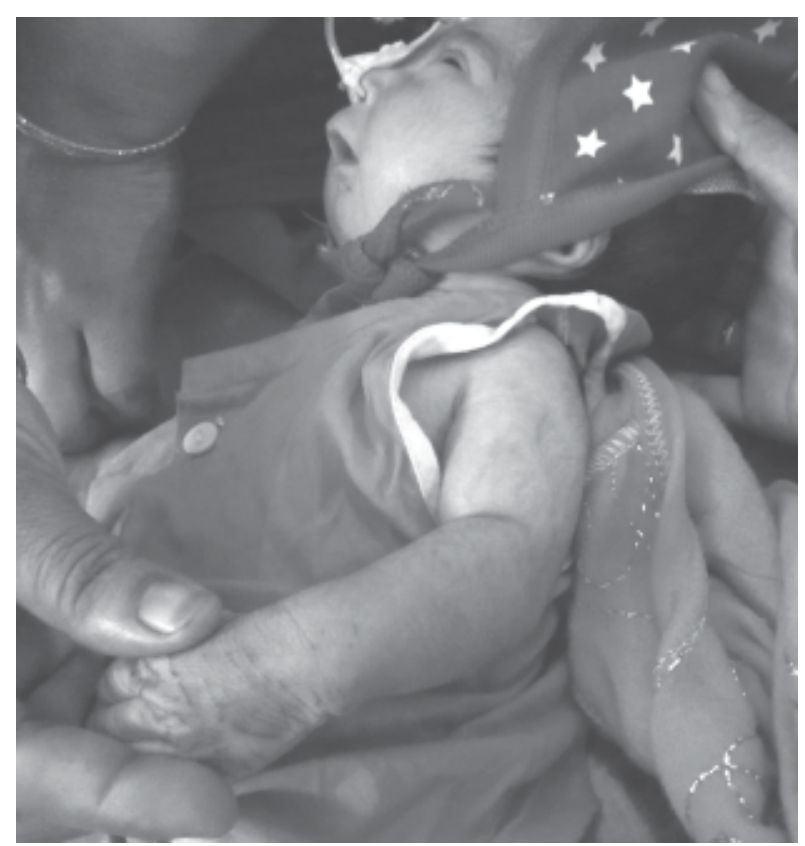

Fig.-1: Photograph showing micrognathia, small pinched nose, flat philtrum and limited ability to extend left elbow.

Capillary refill time was 3 second. Heart rate was 160/ min. Respiratory rate was $56 / \mathrm{min}, 1^{\text {st }}$ and $2^{\text {nd }}$ heart sound were normal and murmur was absent. Abdomen was soft and there was no organomegally. Moro reflex could not be elicited because of limited extension of left elbow joint and short lower limbs with bowing of their proximal segments (Fig-2). We diagnosed the case as Infant of diabetic mother (IDM) with multiple congenital anomalies.

On investigation, Hemoglobin was $17.3 \mathrm{gm} / \mathrm{dl}$, platelet count was $1,55,000 / \mathrm{cmm}$, WBC was 32,500 , neutrophil was $84 \%$, lymphocyte was $10 \%$ and CRP was positive. Blood culture showed no growth of microorganism. Ultrasonography of abdomen and brain revealed no abnormality. X ray chest was normal but X-ray of lower limbs showed hypoplasia of femur. $X$ ray of upper limb showed distal radiounar synostosis and separation of proximal ends of radius and ulna (Fig-4).Doppler Echocardiography showed Patent ductus arteriosus. Finally the patient was diagnosed as Femoral hypoplasia with unusual facies syndrome. 


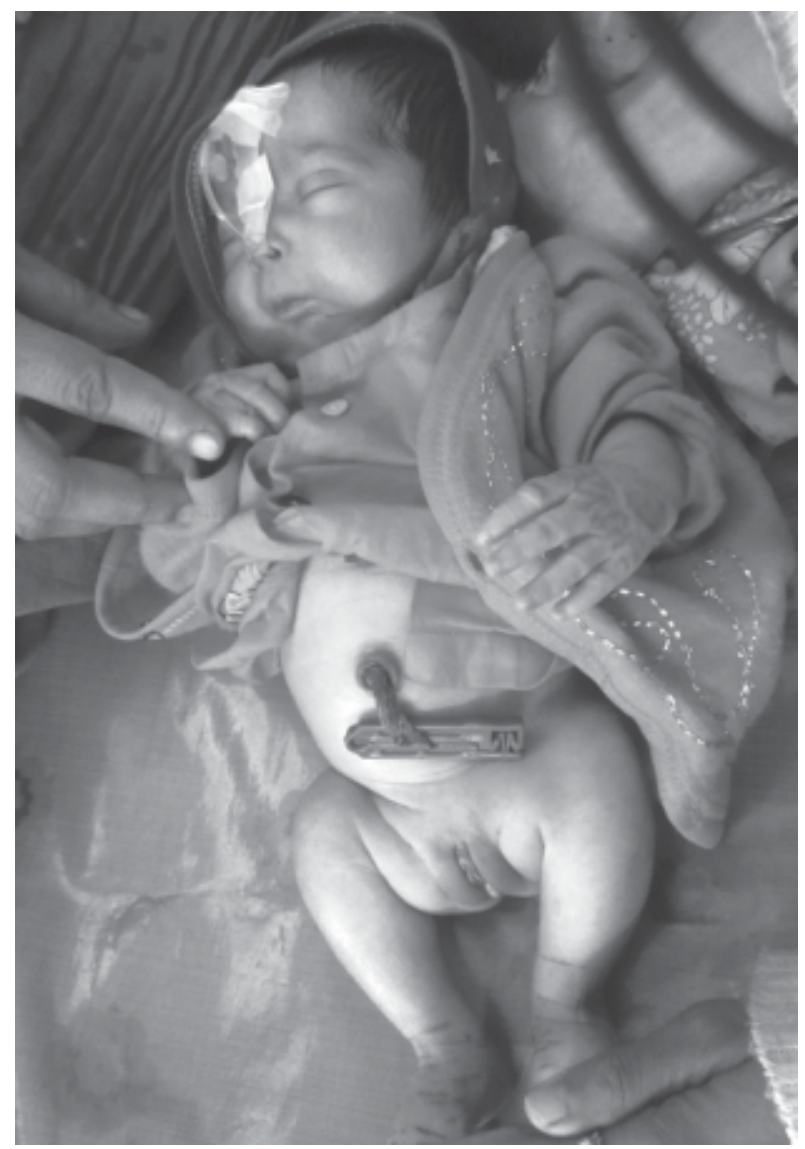

Fig.-2: The proband: Clinical profile

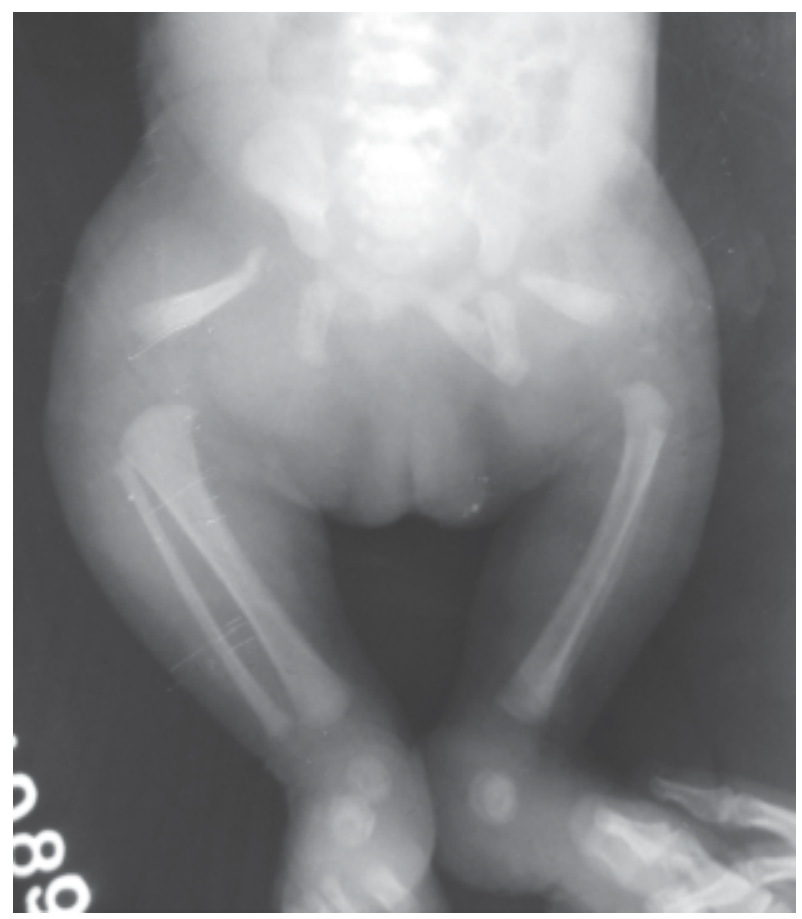

Fig.-3: $X$-ray of pelvis and lower limbs showing femoral hypoplasia

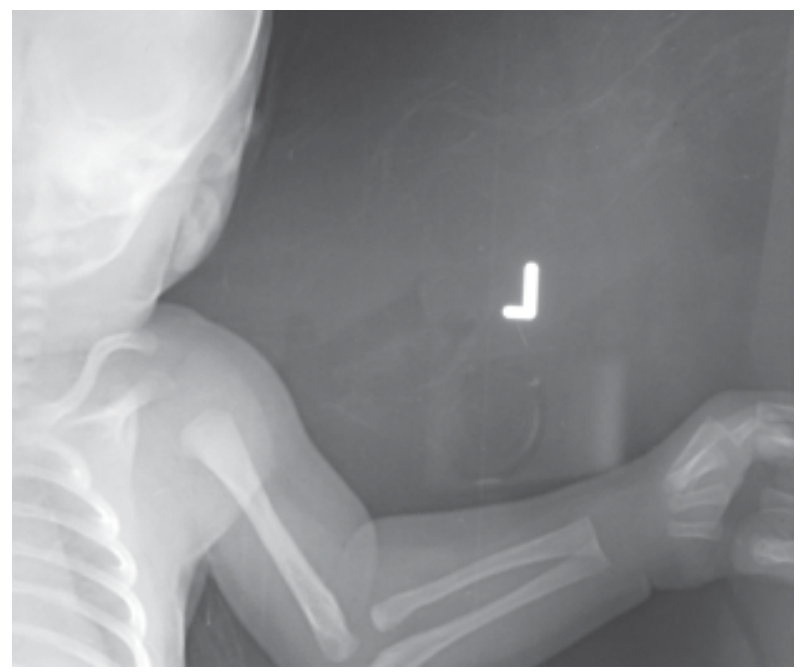

Fig - 4: X-ray of left upper limb and elbow joint showing distal radioulnar synostosis and separation of proximal ends of radius and ulna

\section{Discussion:}

This syndrome includes bilateral femoral hypoplasia, facial dysmorphism with cleft palate, micrognathia, long philtrum, thin upper lip with short broad tipped nose and dysplasia of hips. In addition to craniofacial and skeletal abnormalities other visceral abnormalities in cardiovascular and genitourinary systems may be found. Absence of the femur is a rare finding in $\mathrm{FFS}^{5}$ but radioulnar synostosis as in our case with femoral hypoplasia is another uncommon finding.

Maternal diabetes is known to have teratogenic effects. Malformation including neural tube defects, caudal dysgenesis, vertebral defects, congenital heart defects, femoral hypoplasia and renal anomalies are described in infants of diabetic mothers. However craniofacial anomalies have been rarely reported in such infant. ${ }^{6}$

The caudal dysplasia syndrome and FH-UFS have been reported to be more frequent among infants of diabetic mothers. ${ }^{7}$ Sironomelia or caudal dysgenesis is a syndrome in which there is insufficient mesoderm in the caudalmost region of the embryo resulting in abnormalities of lower limbs and urogenital system but craniofacial anomalies are always absent. The presence of characteristic facial defect with cleft palate together with cardiovascular and genitourinary anomalies have sharply contrasted this case of $\mathrm{FH}$ UFS with sironomelia.

This syndrome may be attributed to fetal constraint, secondary to oligohydromnios. ${ }^{8}$ Our case had 
bilateral femoral hypoplasia, micrognathia, upper thin lip, cleft palate and congenital heart disease like patent ductus arteriosus. With this framework the case fits into classical clinical spectrum of FH-UFS. The finding of flexion deformity of elbow with skin dimpling is reported before 8 but surprisingly radioulnar synostosis with separation of proximal ends is not yet reported. Regarding the outcome there may be problems in speech development but many patients have been of normal intelligence and most of them have been ambulatory.

\section{Conclusion:}

Unusual feature like distal radioulnar synostosis with proximal radioulnar separation may emerge as a part of the spectrum of Femoral hypoplasia- unusual facies syndrome.

\section{References:}

1. Pitt DB, Findlay II, Cole WG, Rogers JG. Case report: Femoral hypoplasia- unusual facies syndrome. Aust Pediatr J 1982; 18: 63-66.

2. Johnson JP. Carey JC, Gooch WM, Petersen J, Beattie JF . Femoral hypoplasia- unusual facies syndrome in infants of diabetic mothers. J Pediatr 1983; $102: 866-72$.
3. Daentl DL, Smith DW, Scott Cl, Hall BD, Gooding CA. Femoral hypoplasia-unusual facies syndrome. J Pediatr 1975; 86: 107-11.

4. Riedl F, Froster- Iskenius U. Caudal dysplasia and femoral hypoplasia- unusual facies syndrome: different manifestations of the same disorder? European Journal of Paediatrics 1985; 144: 80-82.

5. Trabelsi M, Monastiri K, Hammami A, Touzi C, Ben Hariz M, Gharbi HA, Bennaceur B. Hypoplasie femorale avec dysmorphie faciale. Apropos d"une nouvelle observation. Annales de Pediatrie (Paris) 1989; 36: 693-96.

6. Gleiser S, Weaver DD, Escobar V, Nicholos G, Escobedo M. Femoral hypoplasia- unusual facies syndrome from another view point. Eur J Pediatr 1978; 128: 1-5.

7. Ewart Toland A, Yanko witz J, Winder A, Imagire $R$, Cox VA, et al. Oculoauriculovertebral anomalies in children of diabetic mothers. American journal of Medical Genetics 2000; 90: 303-09.

8. Burn J, Winter RM, Baraitser M, Hall CM, Fixsen $\mathrm{J}$. The femoral hypoplasia- unusual facies syndrome. Journal of Medical Genetics 1984; 21: 331-40. 